\title{
VOCES DE LA POESÍA CRÍTICA FEMENINA DE URUGUAY Y DE BRASIL: IDEA VILARIÑO Y HELENA KOLODY
}

\author{
CRISTIAN JAVIER LOPEZ (UVIGO) ${ }^{1}$
}

\begin{abstract}
RESUMEN: Las voces femeninas se encuentran en el canon literario del continente americano en una notoria desventaja en comparación a los escritores, quienes siempre dominaron el campo de las letras. Históricamente la figura de la escritora fue relegada a los márgenes por diferentes motivos de índole social, económica, cultural, etc. A comienzos del siglo pasado, y en ese marco comentado antes, dos mujeres sudamericanas alzaron sus voces de manera contundente: Idea Vilariño, de Uruguay, y Helena Kolody, de Brasil. Ambas autoras exponen en sus obras líricas visiones sobre el conflicto bélico de la Segunda Guerra Mundial y su preocupación con la sociedad de su tiempo. Los poemas de estas dos escritoras demuestran el comprometimiento de la mujer con la sociedad y la consciencia de retratar lo vivido, por medio de la palabra escrita. Revelar ese comprometimiento de las poetas es nuestro objetivo mayor. Para tanto buscamos apoyo, también, en visiones teóricas como las de D’Onofrio (1978), Paz (1991); Guerra (2004; 2007).
\end{abstract}

PALABRAS CLAVE: Literatura Comparada, Idea Vilariño, Helena Kolody; Lírica latino-americana.

RESUMO: As vozes femininas se encontram no cânone literário do continente americano em uma notória desvantagem em comparação aos escritores, os quais sempre dominaram o campo das letras. Historicamente a figura da escritora foi relegada ás margens por diferentes motivos de cunho social, econômico, cultural, etc. A começos do século passado, e nesse marco comentado antes, duas mulheres sul-americanas alçaram suas vozes de maneira contundente: Idea Vilariño, de Uruguai, e Helena Kolody, do Brasil. Ambas as autoras expõem em suas obras líricas visões sobre o conflito bélico da Segunda Guerra Mundial e sua preocupação com a sociedade do seu tempo. Os poemas de estas duas escritoras demonstram o comprometimento da mulher com a sociedade e a consciência e necessidade de retratar o vivido, por meio da palavra escrita. Revelar esse comprometimento das poetas é nosso objetivo maior. Para tanto buscamos apoio, também, em visões teóricas como as de D’Onofrio (1978), Paz (1991); Guerra (2004; 2007).

PALAVRAS-CHAVE: Literatura Comparada, Idea Vilariño, Helena Kolody; Lírica latinoamericana.

\section{INTRODUCCIÓN}

La historia de la literatura en el continente americano tiene como una de sus características el hecho de que en su constitución existe la mención a pocas figuras femeninas con algún destaque en comparación a los bien reconocidos escritores. Los motivos por los cuales las voces femeninas fueron colocadas al margen obedecen a distintas razones, muchas ya conocidas, como el condicionamiento social, la legitimación de lo masculino por sobre lo femenino, el nivel económico, la falta de acceso al mundo de las letras, entre otros.

En este sentido, la estudiosa Lucía Guerra, en la obra Mujer y Escritura - Fundamentos teóricos de la crítica feminista (2007), comenta que

[...] hasta la década de los setenta existió en la cultura de Occidente un prejuicio oculto acerca de la literatura escrita por mujeres. Aparte de las dificultades para publicar, hecho que forzó a algunas escritoras del siglo XIX a utilizar un seudónimo masculino, la crítica no les prestaba mayor atención. Y en caso de que lo hiciera, explicaba y daba un juicio valorativo del texto a partir de una noción estereotípica

\footnotetext{
1 Doutorando em Estudos Literários pela Universidade de Vigo/Espanha; integrante do Grupo de Pesquisa Ressignificações do passado da América: processos de leitura, escrita e tradução de gêneros híbridos de história e ficção: vias para a descolonização, e-mail: cj_lopez2@hotmail.com
} 


\section{$=$ TRAMA $=$}

de lo femenino, destacando aspectos como un estilo sutil y poético, la presentación de conflictos del corazón y el esbozo de los trazos íntimos del alma femenina (GUERRA, 2007, p. 7).

Sin embargo, dentro del marco temporal de comienzos del siglo pasado, dos voces femeninas ganaron reconocimiento, y en el intento de revelarlas se eligieron, para escribir este artículo, dos poemas de esas dos escritoras sudamericanas: la uruguaya Idea Vilariño y la brasileña Helena Kolody. Ambas autoras contribuyeron con la literatura de sus países por medio de un trabajo cuidadoso que se puede observar en su producción lírica. En ella se exploran los elementos fónicos y rítmicos de la lengua, es decir, su musicalidad. Esto ocurre en confluencia con el sentido del texto. Las dos autoras escribieron sobre el contexto social en el que vivieron por medio de un lenguaje diáfano que busca acercar el lector a experiencias cotidianas. En los temas desarrollados por las escritoras vemos que ellas reflexionan, mediante un lenguaje sencillo, la figura del inmigrante, la música como fundamento e inspiración, el paso del tiempo $y$, con destaque, el aspecto seleccionado para este trabajo: la crítica social.

Por un lado, la poeta uruguaya Idea Vilariño fue una mujer actuante en el círculo letrado de su época con fuertes convicciones políticas, además de defender una postura rígida en lo que respecta a la crítica literaria y, específicamente, la crítica al canon literario español hecho que se concreta en los estudios sobre poesía que elabora a lo largo de su carrera. Por otro lado, tenemos la poeta Helena Kolody, de Brasil, quien explora de manera significativa la forma nipona del haikai, que la coloca en su país como la primera mujer, hija de inmigrantes ucranianos, que explota este tipo de construcción lírica.

Ambas escritoras presentan puntos en común como el contacto con las artes en su formación académica y eso se da especialmente con la música. Además, las dos ejercieron la docencia y dominaron, en el ámbito de la lírica, las formas fijas. Sus poemas se fundamentan en las construcciones basadas en la musicalidad de la lengua de sus países, buscando una sonoridad próxima al lenguaje coloquial sin perder el cuidado que el arte, que se vale de la manipulación del lenguaje, requiere. En este sentido, podemos notar que la obra de Vilariño, casi en su totalidad, fue escrita en la variante del español rioplatense. En la obra de Kolody presenciamos el uso del portugués brasileño, cuya sonoridad, distante del portugués europeo, queda explícita en las ricas combinaciones fonéticas que otorgan musicalidad a sus poemas. En este sentido, caben las palabras del estudioso Octavio Paz, quien llama la atención para un punto claro que se debe tener en cuenta al momento de comprender al arte literario creado en el espacio latinoamericano. Según el estudioso mexicano, las obras creadas en este continente

[...] son literaturas escritas en lenguas transplantadas. Las lenguas nacen y crecen en un suelo; las alimenta una historia común. Arrancadas de su suelo natal y de su tradición propia, plantadas en un mundo desconocido [...] se transformaron. Son la misma planta y una planta distinta. Nuestras literaturas no vivieron pasivamente las vicisitudes de las lenguas transplantadas [...] dejaron de ser meros reflejos transatlánticos; a veces han sido la negación de las literaturas europeas y otras, con más frecuencia, su réplica (1991, p. 8).

Así, al tratar sobre las literaturas de América, advenidas de las lenguas heredadas del Viejo Mundo, nos afrontamos con lo que históricamente aconteció en el propio continente durante su conformación territorial, tanto en los aspectos físicos y políticos como en aquellos espacios pertenecientes al campo de las ideas, de la construcción cultural.

En ese sentido, según señala Salvatore D’Onofrio (1978, p. 24): 


\section{$=$ TRAMA $=$}

[...] é preciso salientar ainda que o poeta não cria a língua, pois esta é um código comunitário, mas apenas dela se serve de uma forma diferente. Ademais, ele compartilha com a comunidade em que vive não somente o código linguístico, mas também a ideologia, o cabedal cultural, as características bio-psíquicas. A arte é 'uma construção formal baseada em elementos do mundo real' e, como estes, ela possui a qualidade da estruturação.

Por lo tanto, desde esta perspectiva de lenguas transplantadas se entiende el análisis de este trabajo con poemas de dos escritoras en las variantes de cada una de las lenguas de sus países.

Para la ejemplificación referente al tema de la crítica social que las poetas realizaron, se seleccionaron, para este trabajo, dos poemas ${ }^{2}$ escritos en el marco de un período y hecho histórico específico: La Segunda Guerra Mundial. El primer poema se sitúa en el año de inicio del conflicto bélico y fue escrito por Vilariño en el año de 1939: "No sé que hay en la tarde"3. El segundo poema, de Kolody, se titula "Ampulheta da hora presente"4 y fue escrito al final de la guerra, en el año de 1945.

Ambas producciones forman parte de una serie de poemas en los que las autoras exponen sus perspectivas acerca contexto social-mundial y en las cuales es posible encontrar la voz de la mujer latinoamericana preocupada con la problemática de su tiempo y comprometida con esa misma, exponiendo una conciencia de finitud notoria.

Se espera que el estudio presentado contribuya al conocimiento del aspecto de la crítica social, cultivado en las obras de las escritoras seleccionadas, con el fin de mostrar dos voces femeninas del sur de nuestro continente que se alzaron de modo crítico ante el momento social que las circundaba.

\section{LA VOZ POÉTICA DE LA MUJER LATINOAMERICANA ANTE EL CONFLICTO BÉLICO DE LA SEGUNDA GUERRA MUNDIAL}

Las creaciones poéticas de las autoras Vilariño y Kolody tienen una variedad de temas que van desde el amor, el paso del tiempo, la música, la inmigración y la crítica social. Ambas poetas desarrollaron, desde su lugar de escritoras en sus países, poemas en los que se demuestra el compromiso social de la mujer preocupada con su entorno. Como es de esperar, la producción de cada una de ellas tiene claras marcas que revelan las idiosincrasias de sus países.

Al respecto, cabe destacar la importancia de la variedad de temas cultivados por las poetas, y especialmente el de la crítica social, puesto que históricamente la escritura femenina estuvo cercada por las imposiciones de aquello que era considerado pertinente que una mujer escribiese. En ese sentido Guerra comenta que

[...] la imposición de la maternidad como único rol social o la condena al anonimato son, para las escritoras actuales, eslabones que han dejado de existir. Su escritura, en un nuevo devenir que le permite participar activamente en la cultura, constituye el engendro de otras historias que modificarán, de manera significativa, los rasgos evolutivos del signo mujer [...] (GUERRA, 2004, p. 175).

Así, por un lado, mientras Idea Vilariño llega al círculo social letrado de su país y expone de manera más contundente su pensamiento y posición política, Helena Kolody, que accede al

\footnotetext{
${ }^{2}$ El análisis de los poemas es realizado respetando la escansión que cada lengua exige. Sin embargo, la terminología adoptada para las explicaciones métricas estará basada en las nomenclaturas en lengua española. ${ }^{3}$ Este poema pertenece al poemario Poemas anteriores (2012).

${ }^{4}$ Este poema pertenece al poemario Música submersa ([originalmente lanzado en 1945] 2011).
} 


\title{
$=$ TRAMA $=$
}

círculo de las letras después de conseguir el segundo lugar en la premiación de la Sociedad de Hombres de Rio de Janeiro, cultiva una poesía mucho más centrada en temas etéreos. Sin embargo, existe el punto de confluencia en la producción de estas dos escritoras sudamericanas: la crítica social. En este sentido, la estudiosa Guerra comenta que "en el nuevo discurso crítico latinoamericano, la perspectiva feminista no sólo ha significado el rescate o revaloración de los textos creados por la mujer sino también un serio inquirir en la problemática de la identidad." (2004, p. 174).

Como primer ejemplo está el poema de Idea Vilariño intitulado "No sé qué hay en la tarde", cuya fecha de creación es el año de 1939, inicio de la Segunda Guerra Mundial. En esta producción la poeta uruguaya revela, de forma contundente, la visión de mundo y cuánto esa visión crítica la afectaba como escritora en su realidad sudamericana. La construcción discursiva de ese poema está íntimamente relacionada con la visión existencialista trágica que Idea expresa también en algunos pasajes de su diario íntimo ${ }^{5}$.

La obra lírica se destaca por unir de manera exquisita la forma del poema con el contenido desarrollado en la escritura para dar, mediante esta combinación, un sentido más concreto al contenido existencialista y de crítica que es abordado en esa producción. A continuación está la transcripción del poema de la escritora uruguaya:

\author{
No sé qué hay en la tarde, en la luz, en el alma, \\ no sé si fue esa música dolorosa y fantástica \\ o si es este silencio perfumado y oscuro \\ o esta luz de crepúsculo perfumada y callada. \\ Me faltan tantas cosas que me duelen las manos \\ que se alargan dolientes, pálidas y vacías. \\ Da hasta miedo seguir \\ si con tan pocos años pesa tanto la vida. \\ Nunca tan cerca de la vida. Nunca. \\ Nunca tan grande como hoy la muerte, \\ sobre todo, ante todo, al fin de todo, \\ y yo, sintiéndome ir trágicamente. \\ La tarde que se muere se agiganta. \\ Yo me siento perdida. \\ Da hasta miedo seguir \\ si con tan pocos años pesa tanto la vida. (VILARIÑO, 2012, p. 20).
}

La composición de Idea Vilariño, que está dividida en cuatro cuartetos, presenta un diálogo interesante entre el tema y la métrica adoptados a lo largo del poema. Esta variación métrica de los versos que acompaña el sentido expresado por el yo lírico es vital para entender el mensaje propuesto. De este modo, la poeta explora, de forma instigadora, la relación de los diferentes versos haciendo gala de su dominio sobre la métrica.

El primer cuarteto se despliega como una incógnita del yo lírico que intenta dilucidar el porqué o los motivos de su aflicción. Durante el transcurso de esta estrofa se expone una composición de versos de quince sílabas. La utilización de esta forma composicional, al comienzo, no muestra grandes secretos, sin embargo, durante todo el poema, la poeta presenta, para el lector, una conexión de la estructura métrica del poema con los versos que, a medida que se llega a una evocación más íntima por parte del yo lírico, van disminuyendo las sílabas poéticas. Esta disminución en el metro de los versos puede ser vista como una alusión

\footnotetext{
${ }^{5}$ Idea Vilariño dejó 17 libretas en las que escribió su diario. Parte de ese diario fue lanzado en la obra Diario de Juventud, en el año de 2013, por la editorial Cal y Canto, de Montevideo.
} 


\section{$=$ TRAMA $=$}

que hace la poeta a la situación de continuidad del tiempo, un fluir que lleva al agotamiento de este y que se concreta en la reducción de sílabas poéticas de la composición.

El verso número uno, de 15 sílabas poéticas, presenta quizás el sentido estructural de la construcción del poema, pues tal verso tiene un carácter que va desde lo general a lo específico. Esto puede ser percibido mediante el uso de los vocablos "tarde", que evoca una apertura del campo de visión, un espacio abierto, que luego dirige la atención para la palabra "luz", es decir, la mirada se centra en el efecto lumínico específico de lo que antes era un campo abarcador para, de esa manera, llegar al punto de introspección mediante la palabra "alma". Esta forma, que va desde lo externo hasta lo interno, será desarrollada en las estrofas posteriores.

Después del primer verso, los siguientes continúan el desarrollo de la incógnita que aflige al yo lírico. El verso segundo comienza repitiendo la misma construcción del anterior reforzando la pregunta o incertidumbre: "No sé". En este caso los elementos lingüísticos utilizados conducen al lector a lo que podría considerarse como una búsqueda en la memoria mediante la evocación de los sentidos como la audición, la visión y el olfato. La audición se revela gracias a la palabra "música", que es adjetivada como "dolorosa" y "fantástica", la visión y el olfato son perceptibles en el tercero y cuarto versos en los que se encuentran las combinaciones substantivo/adjetivos "silencio perfumado y oscuro" y "luz perfumada y callada".

El cuarteto número dos propone una aproximación más concreta al sentimiento de dolor que el yo lírico expresa. Ese acercamiento es posible de ser observado en los versos "Me faltan tantas cosas que me duelen las manos/que se alargan dolientes, pálidas y vacías." Tales expresiones son escritas ahora en versos de catorce sílabas. Al leerlos, el lector se ve frente a una constatación de sufrimiento, ante una angustia de pérdida o de no tener. El uso del encabalgamiento ayuda al entendimiento sobre la idea de alargar o extender ese dolor que el yo lírico expone en estos versos por medio de una frase que métricamente se debe leer en su totalidad.

Existe en uno de los pasajes, que la poeta uruguaya escribe en sus memorias de juventud, una reflexión sobre la vida en la cual expone su conciencia sobre la finitud. En este mismo pasaje, que abajo se trascribe, es constatable la importancia que tiene para Idea Vilariño el acto creador que viene a ser una esperanza para el ser humano:

Pero esta voluntad de muerte, no es un ansia de terminar, como aquella que pueden provocar el dolor o la pasión. -está allí-. Y espera a cumplirse sin desgarrar más a otros. -El momento llegará-, Mientras tanto se puede ir embelleciendo la sala de espera. El crear Belleza es grabar algo de lo poco de valor que nos pasa por las manos. Para nosotros y para los otros. No importa. Para que esté mientras somos (VILARIÑO, 2013, p. 196).

Por otro lado, es interesante verificar que, así como expresa en su diario, la conciencia determinista es traducida para su poesía, explorando esta temática en su universo lírico. La escritora demuestra la gravedad de su visión acerca del mundo generada por el entorno de su existencia. El interés por registrar en sus poemas su sentimiento de finitud. El dolor de la existencia es una manera de expiación que la poeta encuentra en el acto creador. Nótese aquí el destaque que la escritora da para las manos, elemento evocado por el yo lírico en la estrofa comentada anteriormente en la que se lee: "Me faltan tantas cosas que me duelen las manos". Posiblemente esta inconformidad del yo lírico frente a la existencia es plausible de entender como una realización donde convergen escritora y yo lírico en un solo producto, si comparamos el uso del símbolo de las manos presentes tanto en el diario como en el poema analizado. Una 


\section{$=$ TRAMA $=$}

manera que la escritora montevideana encuentra para exteriorizar su desaliento de manera concreta.

El verso que sigue a esta construcción de la segunda estrofa, compuesta de 14 sílabas, es sumamente significativo. Este está escrito en un heptasílabo, es decir, está estructurado con la mitad de las sílabas poéticas de los versos anteriores. Tal reducción puede ser vista como la angustia del yo lírico demostrado en la expresión "Da hasta miedo seguir". Ese verso vital no sigue con la construcción métrica anterior. Esa acción puede ser una señal, o metáfora, del miedo que expresa el yo lírico a continuar viviendo. Esta angustia se revela concretamente en lo expresado por el yo lírico que expone el motivo de desaliento en el verso cuarto de la segunda estrofa: "con tan pocos años pesa tanto la vida". Esta expresión encuentra fuertes ecos en la escritura del Diario de Juventud (2013), de Idea Vilariño en el que aparece la voz femenina; la mujer que sufre.

Este poema cobra un significado todavía más contundente ya que el contexto de su producción es el año en que inicia la Segunda Guerra Mundial. Por lo tanto, el lector está frente a una obra de una joven poeta angustiada por la situación global que el conflicto apuntaba.

Es un contexto de producción marcado por el sufrimiento y el desconcierto por tal hecho histórico, que habría desencadenado el dolor que el yo lírico comenta con tan poca edad ("con tan pocos años pesa tanto la vida"). Si se considera lo escrito por la poeta, fuera del universo lírico -como las manifestaciones en su Diario de Juventud, ya mencionado anteriormente ${ }^{6}$, para entender el proceso creativo, podemos considerar este poema como una de las formas más claras y directas en que la poeta expresa lo más íntimo de su ser frente al mundo que la rodea y que constituye el universo histórico-social en que se inserta su producción.

El tercer cuarteto arriesga a implementar otra disminución del metro, esta vez es un endecasílabo. Como se puede constatar, así como el primer verso del poema va de lo general - como la imagen de una tarde, a lo particular, como el espacio más interior del alma, la disminución de la métrica acompaña el carácter de introspección que el yo lírico busca exponer en la composición.

La disminución del verso gana más sentido con la utilización que hace el yo lírico del adverbio "Nunca" para expresar, de modo categórico, la fatalidad de su aflicción. La angustia de morir aparece explícitamente en este cuarteto. El lenguaje empleado busca, cada vez más, aproximar el lector a la atmósfera de introspección para que él pueda "experimentar" el sentimiento del yo lírico, manifestado en los versos

\footnotetext{
Nunca tan cerca de la vida. Nunca.

Nunca tan grande como hoy la muerte, sobre todo, ante todo, al fin de todo, y yo, sintiéndome ir trágicamente.
}

En esta estrofa el yo lírico retoma lo expresado en el verso anterior acerca de su juventud ("tan pocos años"), para volver a reiterar este tema "cerca de la vida". Es importante destacar que la autora tenía diecinueve años de edad al momento de escribir el poema. El yo lírico en el segundo verso de este cuarteto retruca la juventud con la sensación de muerte en el momento de la enunciación. Esa muerte se encuentra abarcando la totalidad de la existencia "sobre todo", "ante todo" y "al fin de todo". El desasosiego del yo lírico es inexorable y termina en el cuarto verso con la sensación que se concreta de manera trágica.

\footnotetext{
${ }^{6}$ De acuerdo a lo que se registra en el diario de la poeta: "Quiero decir esto: Todo lo que ha plasmado en poesías, todo lo que paso a la libreta de poesías, es lo único que he vivido verdaderamente. Todo lo que yo diga sentir que no esté apoyado por un poema, puede no ser cierto." (Diario, 12 de octubre de 1941], VILARIÑO, 2013, p. 41), que anteriormente transcribimos.
} 


\section{$=$ TRAMA $=$}

El último cuarteto se vale de casi todos los metros utilizados en el poema. Esta estrofa resume, de modo general, los aspectos referentes a la forma y al tema que la escritora busca exponer para el lector.

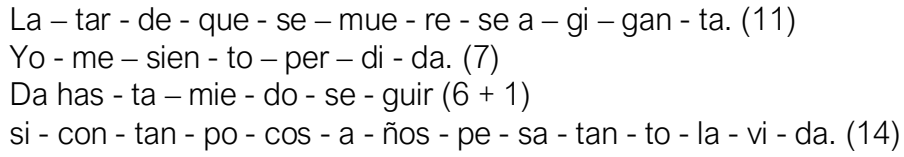

Además es en esta parte del poema, segundo verso exactamente, que se revela el género del yo lírico, femenino: "Yo me siento perdida.". Este dato aparece como relevante debido a que a lo largo de este análisis expusimos de manera conjunta el sentir de la poeta, mediante lo escrito en su diario de juventud, con lo expresado por el yo lírico en el poema. Existe, por lo tanto, la posibilidad de encuentro entre escritora y yo lírico. Idea en esta composición expresa, a la edad de diecinueve años, una preocupación concreta con la conciencia de finitud de la vida. Hecho que se vuelve más significativo cuando lo juntamos al año de producción.

El verso "La tarde que se muere se agiganta." Expone la escena pintada al comienzo del poema. Una amplitud de horizonte y un escenario para la reflexión del yo lírico. En los dos versos siguientes tenemos la expresión del yo lírico -que se manifiesta en el género femeninosobre la sensación de angustia y de desesperación "Yo me siento perdida. / Da hasta miedo seguir". Para terminar con el verso pleno de catorce sílabas resumiendo el tema "si con tan pocos años pesa tanto la vida."

Si se piensa en la posibilidad de una trasmutaciónlconexión entre el Yo lírico de "No sé qué hay en la tarde" y las declaraciones de Idea Vilariño en sus registros personales, entonces es posible verificar que en esta producción hay una síntesis del existir y del concebir la vida por parte de la poeta. De ese modo, se puede ver como ella se vale de la lírica para expresar su subjetividad y exteriorizar, por el arte literario, una visión de mundo que expresa con las palabras: "pesa tanto la vida".

El segundo poema elegido para analizar y exponer la voz femenina de crítica social es el poema "Ampulheta da hora presente" (KOLODY, 2011, p. 190). Esta obra, como fue comentado antes, forma parte de una producción lírica especíica de Kolody, en la que la autora revela su lado crítico ante la situación de la guerra. En el poema, la poeta brasileña realiza una exposición de la catástrofe de la guerra y los tristes resultados de ésta.

La obra expone la voz enunciadora de un yo lírico que expresa su preocupación con el presente de la escritora al momento de su creación y con las consecuencias que traen los conflictos armados, como se puede ver a continuación

\section{Ampulheta da hora presente}

Torva, a ampulheta goteja sangue.

A hora terrivel passa, esmagando o coração da humanidade.

Pesada, a angústia escorre na ampulheta... 


\title{
$=$ TRAMA $=$
}

\author{
Contínua, \\ enervante, \\ avassaladora angústia \\ dos que desejariam suster a derrocada \\ e nada podem fazer... \\ E nada podem fazer (KOLODY, 2011, p. 190).
}

Ese poema se basa en, como puede ser observado, uno de los componentes principales de la poética kolodyana: la efemeridad o transitoriedad del tiempo y en él se teje una reflexión sobre la guerra.

La voz femenina se vale de la forma del poema para exponer su perspectiva sobre los lugares comunes como el tiempo y lo bélico. Desde el título de la obra la escritora ofrece al lector, simbólicamente, ese aspecto mencionado al traer la imagen del reloj de arena (ampulheta). Cabe recordar que, aunque es el paso del tiempo que se representa con la imagen del reloj, el poema hace referencia directamente al presente vivido por la escritora en el año de 1945. Este aspecto es constatado en la elección de tiempo verbal que el yo lírico expone a lo largo de la composición, por medio de las palabras como "goteja", "passa", "escorre", "podem".

Es de considerar, en esa elección, una angustia compartida directamente entre el yo lírico y la poeta, teniendo en cuenta que "la hora presente" remete al momento por el cual la sociedad está atravesando con la finalización de la Segunda Guerra y los sentimientos de incertidumbre que, de modo general, se encuentran latentes en las diferentes sociedades.

El poema está estructurado en catorce versos distribuidos en seis partes de métrica variada:10-7-11-11-12-6-6-8-2-3-7-8-7-7. En cada una de las seis partes la escritora destaca un aspecto diferente de la temática desarrollada en el poema. La división de las partes del poema queda expresada de la siguiente manera: 10, 7-11, 11, 12-6, 6-8, 2-3-7-8-7-7. El primer verso está compuesto por diez sílabas poéticas en las que el yo lírico presenta el tiempo presente, caracterizándolo como "torvo". La elección y utilización de este signo lingüístico ofrece una doble interpretación, pues, por un lado, puede expresar terror o pavor e ira o furia al tiempo que ofrece, por otro lado, características de índole pictórica al significar también oscuro y sombrío.

Por lo tanto, es de suponer que la hora presente a la que se refiere el yo lírico carga una connotación altamente negativa que se torna, todavía, más evidente cuando expresa el contenido existente en la "ampulheta". Técnicamente, debería ser arena, sin embargo, en el cuadro pintado en el poema es sangre.

El pareado siguiente, y segunda parte del poema, muestra que el yo lírico expresa el transcurrir del tiempo anteriormente caracterizado como turbio es ahora adjetivado como terrible y alcanza a todos, como sentimiento mundial, de manera sumamente profunda: en el "coração da humanidade". Es interesante verificar que la sensación del presente está expuesta en la acción o movimiento transmitido gracias al uso del gerundio.

La próxima parte del poema es un verso que utiliza una estructura semejante a aquella que la poeta presentó en el primer verso. En ese verso, el yo lírico se refiere a la sangre como angustia, pues ella "escorre na ampulheta". Existe un sentimiento de pesar intenso que se materializa en el uso de la palabra que caracteriza la angustia dentro del reloj de arena: pesada.

La escritora opta por colocar en este verso un signo gráfico efectivo para continuar con la idea de continuidad y movimiento del reloj. El verso termina con puntos suspensivos que, por un lado, suspenden la frase y generan la idea de continuidad* dejando espacio a que el lector complete o continúe el sentido que el yo lírico expresa. Después de este verso se da lugar a los versos que continúan exponiendo las consecuencias de esa hora terrible. 


\section{$=$ TRAMA $=$}

El próximo pareado, cuarta parte, presenta un elemento que la poeta brasileña utilizó como parte de su obra en general, y que cabe mencionar que en el poemario al que pertenece la obra en cuestión se da con mayor intensidad, que es la imagen del niño. Así, el yo lírico utiliza el calificativo de la "injustiça" para describir lo que sucede con las verdaderas víctimas de la catástrofe de la guerra. Ese sentimiento queda explícito, pues los ojos de los niños son "trágicos e surpresos". La figura infantil es de importancia vital, porque hasta este momento el yo lírico comenta sobre la hora presente, la que se plasma en la mirada de los niños que simbolizan el futuro. Por lo tanto, las consecuencias de la hora presente llegan hasta el futuro de manera trágica y sorpresiva.

Es posible entender, de acuerdo con lo expuesto hasta esta parte del estudio, que la autora decidió utilizar versos únicos y pareados para explicar el acontecimiento de la "hora presente". Esa explicación trae la presentación del problema, la caracterización del mismo y sus víctimas. En la secuencia, aparece un pareado, quinta parte de la obra, en la que se va desarrollando la acción del tiempo presente. Así, con el verso "Inquietação crescente" se prepara al lector para el final, que viene de la hora presente de la guerra con aquellos que "ficaram sem destino".

En ese último párrafo existe una presentación del reloj de arena por medio del uso de elementos que van creciendo como si fuese la caída de la arena "Contínua," - verso de dos sílabas -, "enervante," - verso de tres sílabas -, "avassaladora angústia" - verso de siete sílabas -, "dos que desejariam suster a derrocada" - verso de doce sílabas. La imagen de la sangre de las víctimas se muestra en este párrafo que termina con dos frases que se repiten. La primera de ellas como un pensamiento inconcluso "e nada podem fazer..." debido al uso de los punto suspensivos. Ya la segunda y última frase aparece como conclusión o sentencia de aquello que fue expuesto hasta este punto: "E nada podem fazer." Así, la poeta expresa, en palabras, su incapacidad de acción delante de los destrozos causados por el conflicto bélico.

\section{CONCLUSIÓN}

Como es posible verificar en el análisis propuesto para este trabajo, existen en los poemas elegidos una preocupación de cuño existencialista por parte de las poetas que se explica no sólo por formar parte de la poética de ambas, sino por el hecho de ubicarse en el marco temporal de un acontecimiento significativo como la guerra. Así, también, se verifica en las producciones de las escritoras sudamericanas, Vilariño y Kolody, la voz femenina consciente y preocupada con los acontecimientos sociales de su época y la necesidad de expresar lo que ocurre en el momento de producción en sus obras.

Mientras uno de los poemas se inserta al comienzo del conflicto bélico, demostrando un yo lírico en conflicto existencial ante la falta de esperanza, el otro poema reflexiona al final del período de la guerra, exponiendo sus consecuencias y vislumbrando un futuro, no muy alentador.

Se observa en el poema de Idea Vilariño la preocupación con la forma y el sentido, expresamente marcado a lo largo de los versos transcriptos. La utilización de los recursos escriturales en el poema comentado propone llevar al lector a compartir con el yo lírico la angustia de vivir, ya que la vida, es, en definitiva, un fluir rumbo a la finitud. En ese sentido, es importante entender la construcción que la propia escritora utiliza, pues esa es una herramienta eficaz para la exploración de los signos lingüísticos.

En el poema de Kolody se ve de modo más concreto una poeta preocupada con las consecuencias de la guerra y sus víctimas. La poeta brasileña demuestra claramente el impacto de la Segunda Guerra en el pensamiento social. Así, el uso de metáforas en el poema, altamente cargado de signos, es relevante para conseguir los efectos de sentidos propuestos por la autora 
quien elabora, por medio de imágenes, una creación en la que el lector observa por las palabras un cuadro de sufrimiento que es pintado por la poeta.

Ambas poetas, mujeres del sur del continente y de países que comparten una historia cultural como países colonizados, expresan de manera clara su visión de mundo, que lejos de quedarse con la escritura de poemas presuntuosos o melancólicos, expresan concretamente su subjetividad y sus preocupaciones con hechos de gran relevancia, mostrando el comprometimiento social como seres actuantes y pensantes dentro de las estructuras sociales que las rodean, específicamente las décadas de 1930 y de 1940, en las que la voz femenina aun no era escuchada.

Para alcanzar el entendimiento sobre la poética de Idea Vilariño y Helena Kolody es importante el diálogo con el tiempo histórico y las condiciones sociales que las rodeaban que, de una u otra manera, condicionaban su escritura. Encontrar poetas del sur del mundo que expresen su preocupación con los hechos relevantes a nivel global y lo hagan de forma exquisita con la capacidad escritural de ambas es de gran importancia para los estudios que buscan traer aquellas producciones dejadas de lado o tal vez menos estudiadas en el ámbito académico. En este sentido, para ejemplificar de manera breve, el aspecto de la crítica social en la producción de Helena Kolody es una perspectiva prácticamente nula en los estudios sobre la obra de la autora y la obra de Idea Vilariño, que si bien tuvo proyección internacional, no consigue equipararse en difusión con las obras de sus contemporáneos masculinos.

En los poemas seleccionados es posible observar cómo se concilian la forma/estructura versificada con el contenido/desarrollo temático en la producción lírica. Esa simbiosis es capaz de producir sentidos profundos en ambos planos (estructural y discursivo) que se demuestran de manera concreta en los poemas comentados.

\section{REFERENCIAS}

D'ONOFRIO, S. Poemas e Narrativas: estruturas. São Paulo: Duas cidades, 1978.

GUERRA, L. La mujer fragmentada: Historias de un signo. Chile: Ed. Cuarto propio, 2004. de México, 2007. Mujer y escritura - fundamentos teóricos de la crítica feminista. México: Universidad Autónoma

KOLODY, H. Infinita Sinfonia. WOELLNER, Adélia Maria (Org.) Curitiba, PR: Edição do autor, 2011.

PAZ, O. Convergencias. Barcelona: Seix Barral, 1991.

VILARIÑO, I. Poesía Completa. Montevideo: Cal y Canto, 2012. Diario de juventud. Montevideo: Cal y Canto, 2013. 